\title{
Epigenetics at the base of alternative splicing changes that promote colorectal cancer
}

\begin{abstract}
Alberto R. Kornblihtt Neurociencias (IFIBYNE), Buenos Aires, Argentina.

Chromatin modification influences gene expression by either repressing or activating genes, depending on the specific histone mark. Chromatin structure can also influence alternative splicing of transcripts; however, the mechanisms by which epigenetic marks influence splicing are poorly understood. A report in the current issue of the $\mathrm{JCI}$ highlights the biological importance of the coordinated control of alternative pre-mRNA splicing by chromatin structure and transcriptional elongation. Yuan et al. found that mutation of the histone methyl transferase SEDT2 affects alternative splicing fates of several key regulatory genes, including those involved in Wnt signaling. As a consequence, loss of SEDT2 in the intestine aggravated Wnt/ $\beta$-catenin signaling effects, thereby leading to colorectal cancer.
\end{abstract}

Universidad de Buenos Aires (UBA), Facultad de Ciencias Exactas y Naturales, Departamento de Fisiología, Biología Molecular y Celular and CONICET-UBA, Instituto de Fisiología, Biología Molecular y

\section{One gene, multiple products}

Human genes, as well as those of most multicellular eukaryotes, are organized as mosaics of exons and introns. RNA polymerase II (RNAPII) transcribes the DNA sequence of each gene to generate a precursor mRNA molecule (pre-mRNA) that contains both exons and introns and serves as the substrate for splicing, the process by which introns are removed and exons are joined, yielding the mature mRNA that will eventually be translated to protein. One of the evolutionary advantages of premRNA splicing is the ability to alter mature mRNA via alternative splicing, which allows generation of multiple mRNA and protein variants from each gene, thereby greatly expanding the coding capacity of the genome. Indeed, the number of proteincoding genes in humans is not radically different from that in the worm Caenorhabditis elegans ( 20,000). However, alternative splicing is prevalent in vertebrates (1), strongly suggesting a key role in the higher complexity of these organisms. As a compelling example, alternative splicing occurs in more than $95 \%$ of mammalian genes
(2). In alternative splicing, a single type of pre-mRNA can be spliced in different ways, leading to similar but not identical mature mRNA species. A simple metaphor is that of a textile industry (transcription) that generates many copies of a fabric piece of the same length and quality (pre-mRNAs) and the tailor (the spliceosome) who makes different suits (mRNAs) with each piece of fabric, depending on where he/she cuts and sews (splicing) and on the remnants (introns) that are discarded.

Splicing is carried out by the spliceosome, a multimolecular complex composed of dozens of proteins and ribonucleoproteins. A single spliceosome assembles at every intron to be excised on each premRNA as soon as it emerges from RNAPII during transcription (3). Indeed, accumulating evidence indicates that splicing, or at least the binding of spliceosome and splicing factors to the pre-mRNA that enables it, occurs cotranscriptionally (4). In turn, this cotranscriptionality allows for a complex molecular interplay between the transcription and splicing machineries so that both processes are mutually coordinated and the
Related Article: p. 3375 kinetics and molecular mechanisms intrinsic to each of them influence each other. As a consequence of this coupling, splicing, like transcription, is highly regulated by chromatin structure and the quality and distribution of posttranslational histone modifications (histone marks).

\section{Two models}

Two nonmutually exclusive models have been proposed for the role of chromatin in alternative splicing. In the transcription kinetics model, the preferential positioning of nucleosomes in exons $(5,6)$ or deployment of specific histone marks within the gene body hinder or facilitate RNAPII progression. Changes in overall elongation rates of RNAPII have been shown to promote or inhibit the inclusion of alternative exons in the mature mRNA, depending on the identity of the particular alternative splicing event (7-9). Intragenic histone modifications, including H2B monoubiquitylation (10), H3K9 acetylation and methylation $(11,12)$, and $\mathrm{H} 3 \mathrm{~K} 4$ trimethylation (13), that affect nucleosome organization have been shown to correlate with changes in elongation. Independently of their role in elongation, certain histone modifications, such as H3K79me1, H2BK5me1, H3K27me1, H3K27me2, and H3K27me3, are more abundant throughout internal exons compared with introns and clearly correlate to exon expression (see ref. 14 for a review). In the second model, histone marks are thought to affect alternative splicing by recruiting splicing factors through chromatin-binding proteins (15). For example, H3K36me3 has been shown to recruit MRG15, a chromodomaincontaining protein that is part of several histone-modifying complexes. MRG15, in turn, drafts the splicing factor polypyrimidine tract binding protein 1 (PTB), which controls alternative splicing decisions (16). There are many additional examples in the recent literature of histone modificationsplicing factor associations that affect alternative splicing and evidence of a reverse process through which splicing factors and 
A Intestinal cell with Apc mutated

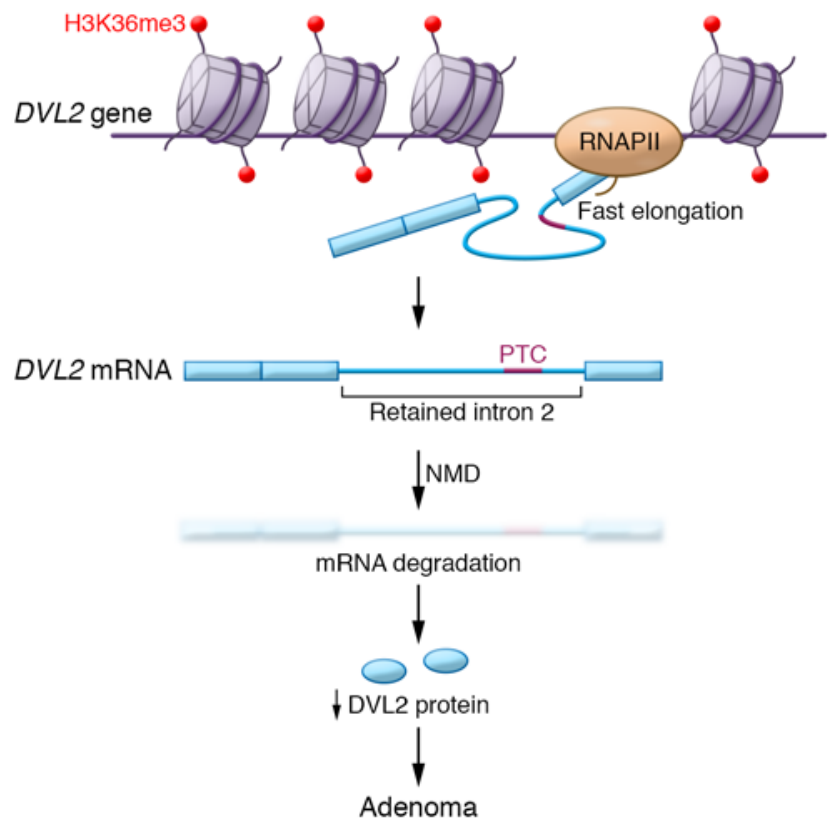

B Intestinal cell with $A p c$ and SEDT2 mutated

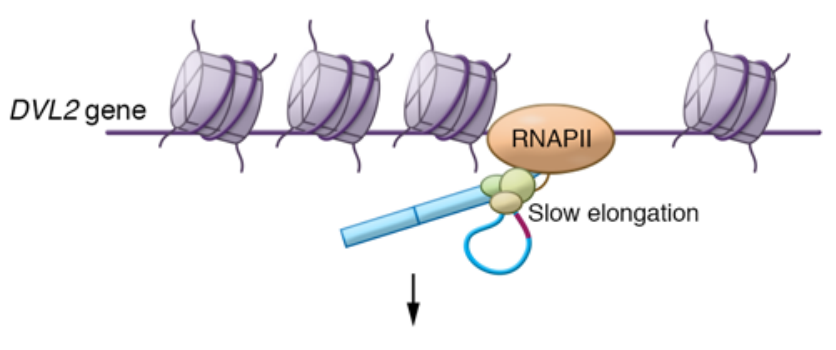

DVL2 mRNA

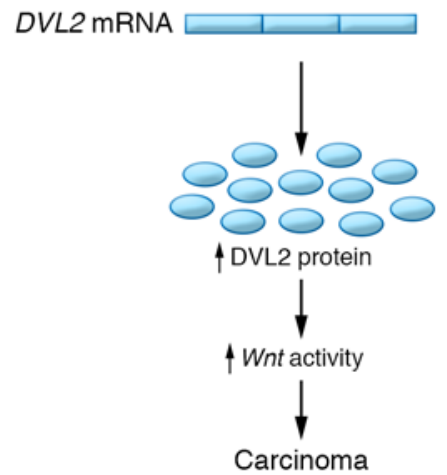

Figure 1. Model for the role of SEDT2 in CRC. Cells in which only the Apc gene is mutated (A) develop adenoma, but have normal levels of H3K36 trimethylation. In the case of the DVL2 gene, normal H3K36me3 levels cause retention of intron 2 in the mature mRNA, promoting degradation of this transcript by NMD as the result of the presence of a premature termination codon (PTC) in intron 2. Subsequent mutation of the SEDT2 gene (B) globally decreases H3K36 trimethylation and reduces intron 2 retention in the DVL2 mRNA. Higher levels of DVL2 protein aggravate Wnt/ $\beta$-catenin-dependent carcinoma progression.

the splicing reaction itself participate in the writing and stabilization of histone marks that affect chromatin structure $(14,15)$.

\section{Colorectal cancer}

Despite an overwhelming accumulation of evidence, there are two persistent questions about the role of chromatin in alternative splicing. One, do changes in chromatin only correlate with changes in splicing, or is there a clear cause and effect relationship between chromatin structure and splicing? Two, most importantly, what are the physiopathological roles of such a complex coupling mechanism? In this issue, Yuan and colleagues (17) present compelling evidence that answers both questions. Specifically, the authors focus on the histone H3K36 methyltransferase SETD2, which has been shown to be mutated in a variety of human tumors. In general, H3K36 methylation is a permissive mark that promotes gene transcription, while repressive marks, such as H3K9me and H3K27me, inhibit transcription. Yuan et al. showed that SEDT2 expression and H3K36 methylation are both downregulated in human colorectal cancer (CRC) tissues compared with normal counterparts. Reduction of
SEDT2 expression in cultured cell lines promoted tumorigenesis, revealing that SEDT2 is a tumor-suppressor gene whose role depends on the catalytic activity of the enzyme. Furthermore, intestinal cellspecific inactivation of the Sedt2 gene in an engineered mouse model surprisingly had no detectable phenotypic or viability effects, suggesting that SEDT2 is dispensable for intestinal physiology. However, in animals harboring one mutated allele of the tumor-suppressor gene $A p c$ ( $A p c^{\text {min/+ }}$ mice), intestinal SEDT2 ablation aggravated the adenoma-promoting role of APC deficiency, significantly shortening life span and greatly increasing the number of CRC tumors. These effects were determined to be the consequence of stimulation of Wnt-dependent transformation and stemness programs, as revealed by the up- and downregulation of the abundance of several signature mRNAs of Wnt signal transduction pathways. Many studies would have stopped at this result and assumed that perturbation of gene homeostasis in the absence of SEDT2 was exclusively due to changes in the transcription of key Wnt genes. However, Yuan and colleagues investigated global changes in
mRNA-splicing variants and discovered more than 700 genes, many of which were syndicated as cancer associated, in the intestines of SEDT2-deficient animals with significant alterations in alternative splicing compared with intestines of control mice. ChIP followed by deep sequencing analyses (ChIP-seq) showed that, upon SEDT2 depletion, there is an outstanding overlap between genes with a reduction of the H3K36me3 mark in their bodies and those with changes in alternative splicing. Moreover, a subset of these genes displayed intragenic enrichment of RNAPII at alternative splicing sites, consistent with a reduction in RNAPII elongation.

One of the prevalent alternative splicing modes regulated by SEDT2 is intron retention. In particular, retention of intron 2 of the mRNA encoding DVL2 decreases with SEDT2 ablation. DVL2 is a member of the dishevelled (dsh) protein family, which is a key component of Wnt pathway signaling. This increase in DVL2's intron 2 excision is presumably a consequence of reduced H3K36me3 around intron 2, which slows RNAPII elongation. As intron 2 contains a premature termination codon of translation, the retention of this intron in DVL2 mRNA 
triggers degradation through a mechanism known as nonsense-mediated mRNA decay (NMD) in WT cells. Therefore, retention of intron 2 in normal cells reduces DVL2 levels, whereas removal of intron 2 in CRC cell precursors, through the epigenetic mechanism elucidated by Yuan et al. (17), increased DVL2 levels, augmenting Wnt signaling and leading to malignant transformation (Figure 1).

\section{Conclusions}

Together, the results of this study highlight the importance of the coupling among the deployment of specific histone marks, alternative splicing regulation, and cell fate. Interestingly, another histone methyl transferase, G9a, that is specific for dimethylation of $\mathrm{H} 3 \mathrm{~K} 9$ has recently been shown to participate in an alternative splicing positive loop that enhances neuron differentiation (18). It is now more evident that when studying the molecular bases of cancer, the view should not be restricted to transcriptional regulation or to epigenetic alterations at the promoter and regulatory regions of genes. Instead, the scope must be widened to fully see how epigenetic changes within gene bodies regulate splicing decisions.

\section{Acknowledgments}

The author is a Senior International Research Scholar of the Howard Hughes
Medical Institute and his laboratory is supported by the Agencia Nacional de Promoción Científica y Tecnológica of Argentina, UBA, and the Consejo Nacional de Investigaciones Científicas y Técnicas of Argentina (CONICET).

Address correspondence to: Alberto R. Kornblihtt, Ciudad Universitaria, Pabellón IFIBYNE. (1428) Buenos Aires, Argentina. Phone: 54.11.45763386; Email: ark@fbmc. fcen.uba.ar.

1. Keren H, Lev-Maor G, Ast G. Alternative splicing and evolution: diversification, exon definition and function. Nat Rev Genet. 2010;11(5):345-355.

2. Barash Y, et al. Deciphering the splicing code. Nature. 2010;465(7294):53-59.

3. Oesterreich FC, Herzel L, Straube K, Hujer K, Howard J, Neugebauer KM. Splicing of nascent RNA coincides with intron exit from RNA polymerase II. Cell. 2016;165(2):372-381.

4. Tilgner $\mathrm{H}$, et al. Deep sequencing of subcellular RNA fractions shows splicing to be predominantly co-transcriptional in the human genome but inefficient for lncRNAs. Genome Res. 2012;22(9):1616-1625.

5. Tilgner $\mathrm{H}$, et al. Nucleosome positioning as a determinant of exon recognition. Nat Struct Mol Biol. 2009;16(9):996-1001.

6. Schwartz S, Meshorer E, Ast G. Chromatin organization marks exon-intron structure. Nat Struct Mol Biol. 2009;16(9):990-995.

7. de la Mata M, et al. A slow RNA polymerase II affects alternative splicing in vivo. Mol Cell. 2003;12(2):525-532.

8. Dujardin G, et al. How slow RNA polymerase II elongation favors alternative exon skipping. Mol Cell. 2014;54(4):683-690.

9. Fong N, et al. Pre-mRNA splicing is facilitated by an optimal RNA polymerase II elongation rate. Genes Dev. 2014;28(23):2663-2676.

10. Fuchs G, Hollander D, Voichek Y, Ast G, Oren M. Cotranscriptional histone $\mathrm{H} 2 \mathrm{~B}$ monoubiquitylation is tightly coupled with RNA polymerase II elongation rate. Genome Res. 2014;24(10):1572-1583.

11. Schor IE, Rascovan N, Pelisch F, Alló M, Kornblihtt AR. Neuronal cell depolarization induces intragenic chromatin modifications affecting NCAM alternative splicing. Proc Natl Acad Sci US A. 2009;106(11):4325-4330.

12. Schor IE, Fiszbein A, Petrillo E, Kornblihtt AR. Intragenic epigenetic changes modulate NCAM alternative splicing in neuronal differentiation. EMBO J. 2013;32(16):2264-2274.

13. Sims RJ 3rd, et al. Recognition of trimethylated histone $\mathrm{H} 3$ lysine 4 facilitates the recruitment of transcription postinitiation factors and premRNA splicing. Mol Cell. 2007;28(4):665-676.

14. Naftelberg S, Schor IE, Ast G, Kornblihtt AR. Regulation of alternative splicing through coupling with transcription and chromatin structure. Annu Rev Biochem. 2015;84:165-198.

15. Luco RF, Allo M, Schor IE, Kornblihtt AR, Misteli T. Epigenetics in alternative pre-mRNA splicing. Cell. 2011;144(1):16-26.

16. Luco RF, Pan Q, Tominaga K, Blencowe BJ, Pereira-Smith OM, Misteli T. Regulation of alternative splicing by histone modifications. Science. 2010;327(5968):996-1000.

17. Yuan $\mathrm{H}$, et al. Histone methyltransferase SETD2 modulates alternative splicing to inhibit intestinal tumorigenesis. J Clin Invest. 2017;127(9):3375-3391.

18. Fiszbein A, et al. Alternative splicing of G9a regulates neuronal differentiation. Cell Rep. 2016;14(12):2797-2808. 